\title{
Experimental Assessment of Low Noise Landing Gear Component Design
}

\author{
Werner Dobrzynski ${ }^{1}$ \\ Deutsches Zentrum für Luft- und Raumfahrt (DLR), 38108 Braunschweig, Germany \\ Leung Choi Chow ${ }^{2}$ \\ Airbus, Filton, Bristol, BS99 7AR, Great Britain \\ Malcolm Smith ${ }^{3}$ \\ ISVR, University of Southampton, Highfield Hampshire, Great Britain \\ Antoine Boillot ${ }^{4}$ \\ Messier-Dowty SA, 78142 Velizy Villacoublay, France \\ Olivier Dereure ${ }^{5}$ \\ Messier-Bugatti SA, 78141 Velizy Villacoublay, France \\ and \\ Nicolas Molin 6 \\ Airbus, 31060 Toulouse, France
}

\begin{abstract}
Landing gear related airframe noise is one of the dominant aircraft noise components at approach. It therefore is essential to particularly reduce landing gear noise. In the European SILENCER project, advanced low noise gears had been designed and tested at full scale. In the current European co-financed project TIMPAN (Technologies to IMProve Airframe Noise) still more advanced low noise design concepts were investigated and noise tested on a $1 / 4$ scaled main landing gear model in the German-Dutch Wind Tunnel. A variety of gear configurations were tested including a new side-stay design, different modifications of bogie inclination, wheel spacing, bogie fairings with different flow transparency, leg-door configurations and brake fairings. The acquired farfield noise data are compared against the results from a landing gear noise prediction model, transposed to full scale flight conditions and compared against the full scale test data obtained for the SILENCER advanced A340 style 4wheel main landing gear. An optimal combination of tested gear modifications led to a noise reduction of up to $8 \mathrm{~dB}(\mathrm{~A})$ in terms of overall $A$-weighted noise levels relative to the SILENCER reference gear configuration.
\end{abstract}

\section{Introduction}

$\mathrm{D}$ ue to the advances in aircraft engine noise reduction, airframe noise became a major noise component during approach and landing. For wide body aircraft in particular the dominant airframe noise sources are the landing gears followed by aerodynamic noise originating from deployed high-lift devices.

\footnotetext{
${ }^{1}$ Research Engineer, Institute of Aerodynamics and Flow Technology, Lilienthalplatz 7, 38108 Braunschweig, Germany.

${ }^{2}$ Engineer, Aerodynamics Department, Building 09B, Filton, Bristol, England BS99 7AR, Great Britain.

${ }^{3}$ Research Engineer, University of Southampton, Highfield Hamphire, Great Britain.

${ }^{4}$ Research Engineer, R\&T Department, Zone Aéronautique Louis Breguet, 78142 Vélizy-Villacoublay, France

${ }^{5}$ Research Engineer, R\&T Department, Zone Aéronautique Louis Breguet, 78141 Vélizy-Villacoublay, France

${ }^{6}$ Research Engineer, Acoustics and Environment Department, 316 route de Bayonne, 31060 Toulouse, France 
Accordingly numerous research efforts were made to reduce landing gear noise either through dedicated wind tunnel experiments or flight tests [1, 2]. Initial noise reduction solutions were the application of solid add-on fairings to protect the complex landing gear structure from the flow [3 - 6]. Based on the results of these experiments it was realized that flow displacement by such fairings could be detrimental regarding additional noise originating from gear components adjacent to those which were faired. As a solution of this problem porous fairings were developed, which would reduce the magnitude of flow displacement but still result in a sufficiently low wake flow velocity not to generate high interaction noise levels with the downstream gear components [7 - 9].

While add-on solutions could be applied at short terms for current aircraft it was realized that low noise gears for future aircraft can best be developed by accounting for noise aspects already in the design stage. A corresponding effort was undertaken in the European research project SILENCER ("Significantly Lower Community Exposure to Aircraft Noise”) [10].

Based on the results of this study, a combination of low noise gear component design and the application of porous fairings [7] was considered to exploit the maximum possible noise reduction potential. This work was performed in an European co-financed research project entitled "Technologies to IMProve Airframe №ise” (TIMPAN) with partners from European aircraft industries, research establishments and academia and focused on an A340 style 4-wheel main landing gear which was originally used in SILENCER, but only limited noise reduction had been achieved accompanied by some weight penalty.

The objective of the TIMPAN study therefore was to develop operational low noise main landing gear components without weight penalties, taking into account modifications in the gear architecture (e.g. wheel spacing and bogie angle) and to optimize and quantify the benefit from the application of porous fairings for various gear components. As in SILENCER the design was based on the relevant constraints predefined by gear functionality and safety for real aircraft application.

\section{Main Landing Gear Configurations}

The design work was based on the SILENCER advanced A340 type 4-wheel main landing gear configuration and focused on

- a low noise design of individual gear components known to represent major noise contributors (e.g. side-stay, various links, leg-door structure and brakes) and

- the noise-wise optimal arrangement of gear components to minimize the interaction of high speed turbulent inflow with complex gear structures (e.g. variation of bogie angle, wheel spacing, placement of fairings and additional ramp door).

Fig. 1 presents a comparison between the SILENCER reference configuration and one of the TIMPAN configurations to better understand the design philosophy in TIMPAN. One of the drawbacks of the SILENCER design was the excessive weight of the telescopic side-stay which, however, allowed for a noise-wise optimal design of the leg-door structure. In TIMPAN therefore a new side-stay design also required a new low noise leg-door design. As shown in Fig. 1 this is a door which is articulated in a way to (once the gear is deployed) protect the complex leg/ drag stay structure from the high speed inflow. It should also be noted (Fig. 1) that for both the SILENCER and the TIMPAN gear design the torque link is installed in front of the leg and is protected through a fairing, while in the back only
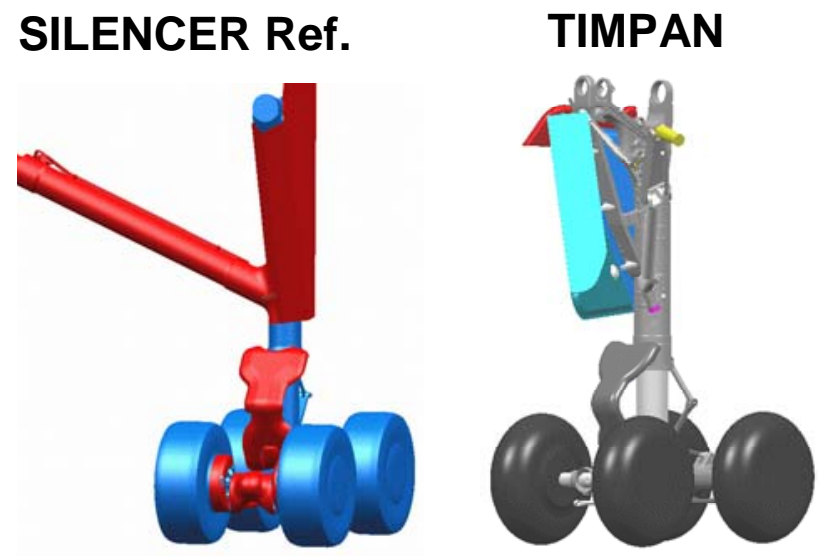

Figure 1. Comparison of SILENCER and TIMPAN main landing gear concepts a narrow slave link is attached to guide the dressings.

Much effort was directed towards the development of a side-stay which could be almost as quiet as the SILENCER clean circular telescopic stay. The final design is depicted in Fig. 2. Compared with the current A340 design the major advantage is the integration of the down-lock springs into the stay to realize a comparatively “clean” design of the components' outer contours. In addition an upstream ramp was provided to, at the same time, shield the still complex stays’ geometry, the upper leg area and the cavity aperture from the flow. 
Also advanced brake fairings were developed in TIMPAN. The brakes were partly recessed and completely separated from the flow by a streamlined fairing. To allow for the necessary brake cooling a mesh-window was foreseen (Fig. 3).

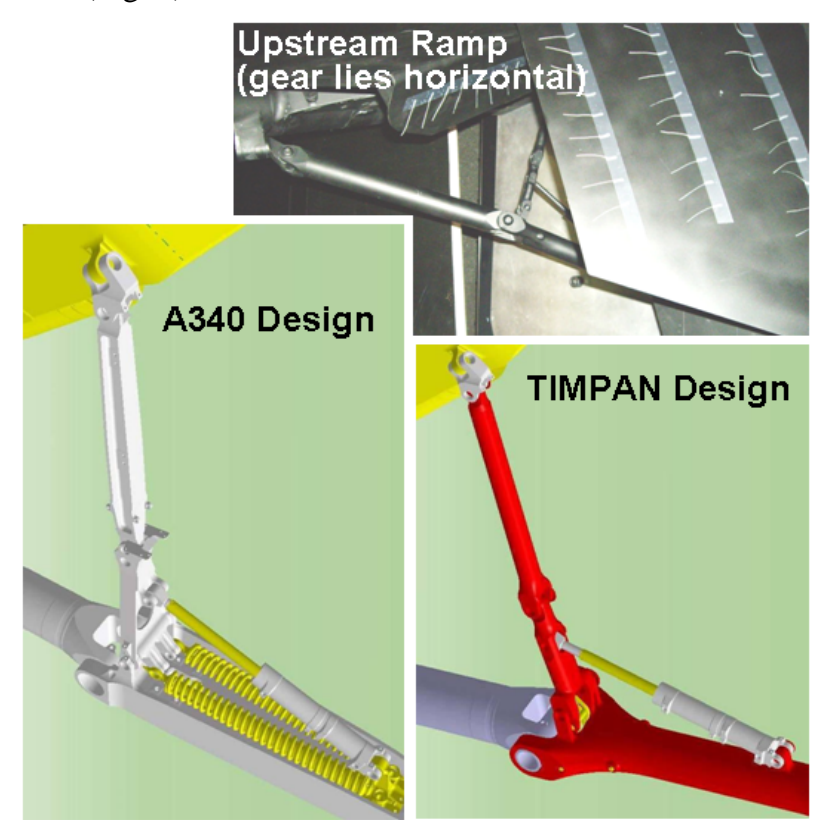

Figure 2. TIMPAN side-stay design and upstream ramp

A low noise arrangement of selected bogie components included:

- Variation of the bogie angle from $0^{\circ}$ (reference) to $15^{\circ}$ toe down.

- Identification of a potentially optimal wheel spacing (Fig. 4) combined with different bogie- and torque link fairings (solid and porous, respectively).

A "narrow" wheel spacing was defined as reduced spacing by $50 \%$ of the tire width relative to the reference total tire spacing and analogous "wide" as a $50 \%$ of tire width increase in wheel spacing.

Examples of the application of solid or porous bogie fairings in combination with a solid or porous torque link fairings are presented in Fig. 5.

Due to budget limitations in TIMPAN only scale model tests were planned in order to make use of the already existing $1 / 4$ scaled SILENCER main landing gear high fidelity mock-up. Accordingly all new gear components were manufactured at that scale to fit to the existing mock-up. The advantage of testing at model scale was that a wide range of configurations could be tested due to correspondingly short stopovers for gear modification.

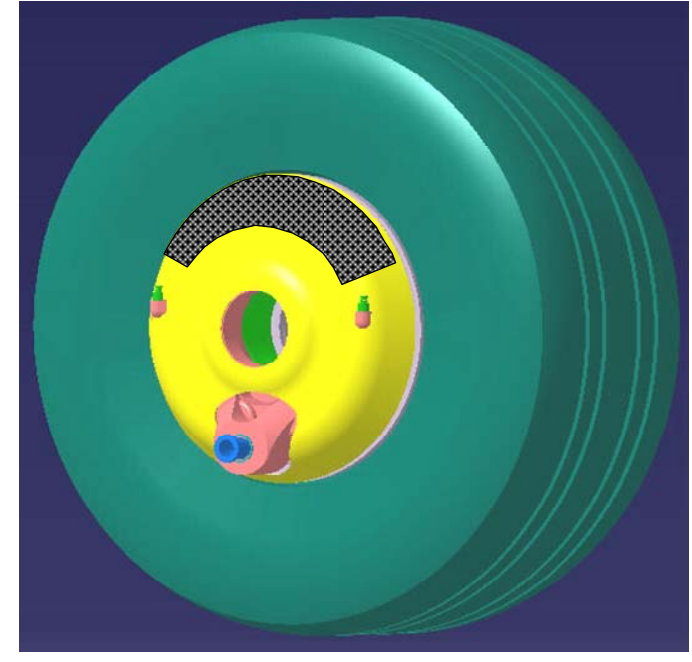

Figure 3. Brake faring with mesh type insert for brake cooling

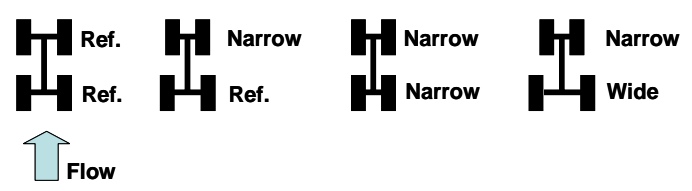

Figure 4. Schematic of selected combinations of forward and rear wheels' spacing
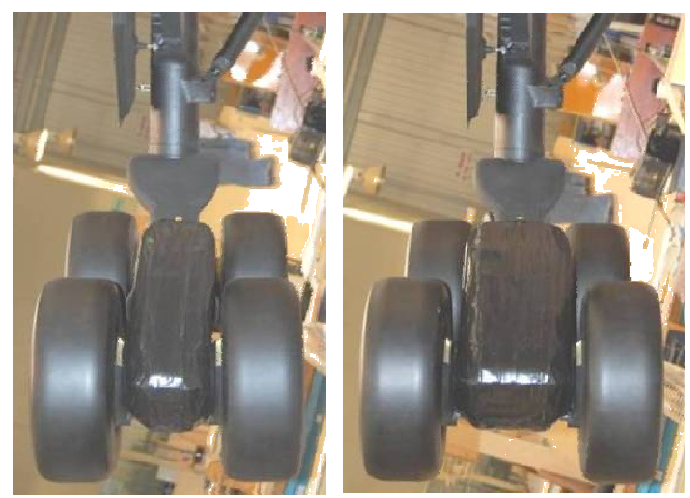

Figure 5. Bogie fairings in combination with torque link fairings for different wheel spacing

\section{Experiments}

Noise measurements were performed in the DNW-LLF (German-Dutch Wind tunnel - Large Low Speed Facility) in its free-jet configuration with a nozzle cross section of $6 \mathrm{~m}$ by $6 \mathrm{~m}$. The maximum wind speed for this tunnel configuration is $78 \mathrm{~m} / \mathrm{s}$ (152 kts), which is close to the typical landing/ approach speed for current commercial aircraft. The anechoic test-hall (the lower limiting frequency is $80 \mathrm{~Hz}$ for broadband noise) allows farfield noise meas- 
urements outside the flow field at lateral distances up to about $18 \mathrm{~m}$ from the very landing gear which is well in both the acoustic and geometric farfield.

\section{A. Wind Tunnel Test Set-up}

As in SILENCER the model gear was installed in a side-wall of $7 \mathrm{~m}$ length, which forms an extension of one side of the wind tunnel nozzle (Fig 6). In $\mathrm{x}$-direction (i.e. streamwise) the gear was installed at a distance of about $5 \mathrm{~m}$ from the nozzle exit plane. The height of the side-wall is $8 \mathrm{~m}$ at the nozzle and $9 \mathrm{~m}$ at its trailing edge (accounting for free jet spreading). Those areas along the wall surface (upper and lower edge areas), which are exposed to the wind tunnel shear layer flow, are treated with absorptive material to minimize flow noise generation and radiation from the side-wall. For the same reason the wall's trailing edge features a saw-tooth shape.

The side-wall arrangement was used to simulate the "in-flight" geometric/acoustic environment (reflection geometry from the wing surface) and to reduce flow noise radiation from the support structure. In order to simulate the actual in-flight lower

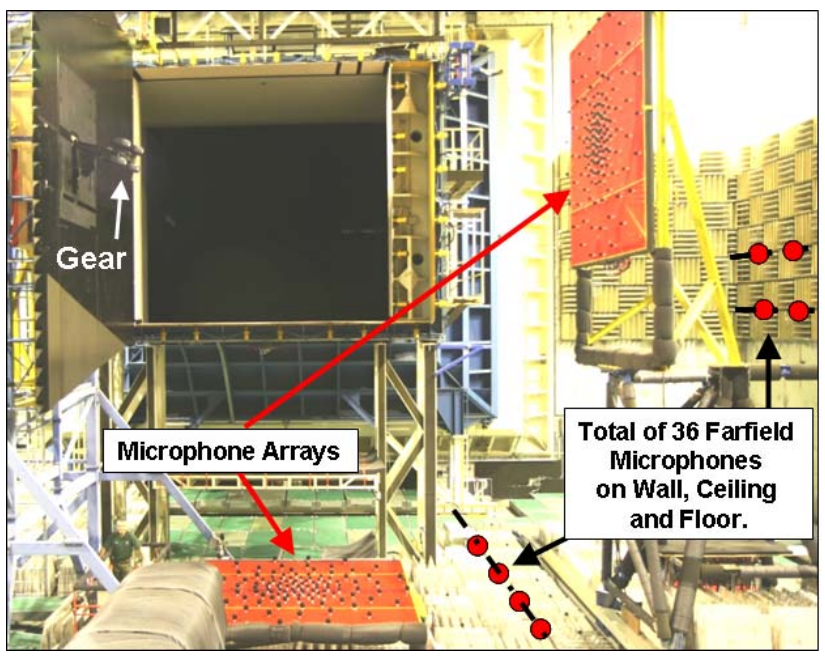

Figure 6. Overview of the measurement set-up in the DNW-LLF $6 \mathrm{~m}$ by $6 \mathrm{~m}$ open test section

wing surface boundary layer thickness the wind tunnel boundary layer was "peeled off” by means of a scoop which is installed along the side-wall's leading edge. In TIMPAN the existing DNW test set up was adapted to install the $1 / 4$ scale mock-up of an A340 type main landing gear for later comparison with the SILENCER WP 2.3 advanced full scale main landing gear test results [10].

During landing/ approach the A340 aircraft typically operates at a characteristic angle-of-attack with respect to the inflow direction. Since in the test set-up the flow direction has to be parallel to the surface of the side-wall this difference between inflow direction and aircraft axis must be accounted for. Based on the gear installation angle in the aircraft (and accounting for deviations of local flow- from flight-directions) a slight backward gear-leg orientation was decided upon for the wind tunnel set-up. On the aircraft the main landing gear-leg is (laterally) inclined with respect to the lower wing surface. Therefore in the test set-up a corresponding inclination angle was realized between the gear-leg and the surface of the side-wall.

The gear bay geometry was not exactly reproduced. Instead an almost rectangular bay was used, internally lined with absorbing foam to avoid acoustic resonance phenomena. However, the bay aperture was exactly simulated.

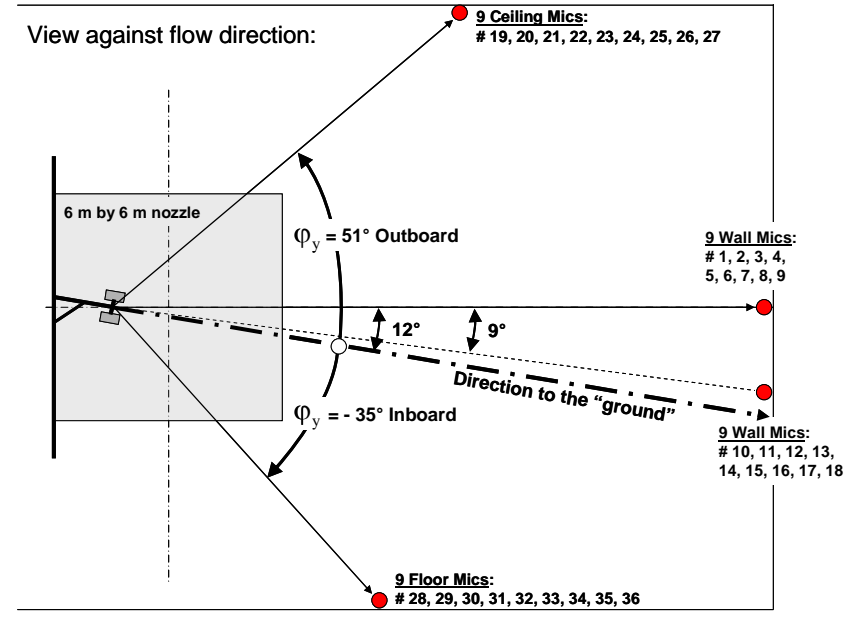

Figure 7. Selected farfield microphone positions on the wind tunnel side wall, the ceiling and the tunnel floor

\section{B. Measurement Techniques and Data Analy-} sis

A similar measurement set-up was applied as in the preceding SILENCER test, i.e. 2 microphone arrays "looking" to the gear from two directions and 4 different rows of microphones (with microphones distributed in flow direction) were installed close to the wall (2 rows), the floor (1 row) and the ceiling (1 row), respectively (Fig. 7). That way noise radiation both towards the "ground" and in sideline directions were determined. In each individual row 9 microphones were positioned at angular increments of about $10^{\circ}$ or less in streamwise direction, covering a range of polar angles between about $60^{\circ}<\varphi_{\mathrm{x}}<125^{\circ}$ (Fig. 7). All farfield measurement positions were equipped with 1/2“-diameter LinearX M51 type electret 
freefield microphones. Acoustic data were acquired up to a frequency of $40 \mathrm{kHz}$.

The analysis and reduction of farfield noise data aimed at the determination of noise level spectra and radiation directivities for different landing gear configurations at different flow velocities. If this basic information is at hand, the measured data may ultimately be extrapolated towards the operational conditions as specified for approach noise certification. To obtain the true source characteristics from wind tunnel out-of-flow acquired acoustic data, sound pressure levels have to be corrected for insufficient signal-to-noise ratio, for the effects of shear-layer refraction [11] (including wave convection), microphone directivity, atmospheric absorption [12] and for the effect of convective amplification (assuming dipole type sources). All farfield noise data were normalized towards a constant propagation radius and will be presented in terms of 1/3-oct. band levels.

To visualize local flow conditions at selected gear components tufting tests were performed. Pictures from two different view angles were recorded by means of two video cameras.

\section{Test Results}

To ensure the quality of the data, the test started with a background noise measurement for the clean side-wall, i.e. without gear and closed gear cavity. The test matrix comprised a total number of 47 gear configurations, resulting from different combinations of individual gear component designs. In order to save measurement time, different from the "standard" procedure of testing for all configurations at 3 different speeds, the majority of configurations were tested at 2 speeds only (i.e. 78 and $62.5 \mathrm{~m} / \mathrm{s}$ ).

To enable an extrapolation of noise data towards speeds beyond the measurement range respective scaling laws must be defined. As one result of previous landing gear noise tests, dipole type noise source mechanisms were found to dominate. Therefore the following velocity scaling of levels and frequencies pertain:

$$
\Delta \mathrm{L}=10 \cdot \log \left(\mathrm{v} / \mathrm{v}_{\text {ref }}\right)^{6}
$$

based on an arbitrary reference speed $\mathrm{v}_{\text {ref }}$. From measured frequencies $\mathrm{f}$ and flow velocities $\mathrm{v}$ the relevant nondimensional Strouhal number St can be calculated as

$$
\mathrm{St}=\frac{\mathrm{f} \cdot \mathrm{s}}{\mathrm{v}}=\text { const. }
$$

with s as characteristic length scale or scale factor.

In order to allow for the comparison of earlier results from full scale tests in SILENCER with the current scale model data in TIMPAN the identical length scale s can be applied, but multiplied by the relevant scale factor thus automatically accounting for 4 times higher frequencies in this model experiment.

To finally present source noise level directivities and account for the source size (model scale factor) at the same time, all data will be referenced towards a constant propagation distance $r_{\text {ref }}$ based on spherical sound attenuation relative to the measurement distance $r$ and accounting for the model (source) size through

$$
\mathrm{L}_{\text {ref }}=\mathrm{L}+20 \cdot \log \left(\mathrm{r} / \mathrm{r}_{\text {ref }}\right)+20 \cdot \log \left(\mathrm{s}_{\text {ref }} / \mathrm{s}\right)
$$

The data from microphones at similar streamwise $\left(\varphi_{\mathrm{x}}\right)$ positions but for slightly different azimuthal angles in the range of $3^{\circ}<\varphi_{y}<12^{\circ}$ (corresponding to the two rows of microphones on the test hall wall) were averaged and considered to represent the noise characteristic for radiation direction towards the "ground". This was considered reasonable since the respective level spectra show similar and systematic variations for all tested gear configurations in the order of less than $1 \mathrm{~dB}$.

In order to check the validity of the anticipated scaling laws to account for the effect of flow speed on broadband landing gear noise, in the following spectra are presented in a non-dimensional form based on Equ. (3) to normalize levels and Equ. (2) to calculate Strouhal numbers from measured frequencies.

Prior to any comparison of noise spectra for different gear configurations as studied in TIMPAN it is worthwhile to check how well the noise spectrum for the original SILENCER full scale advanced main landing gear compares to the noise spectrum as obtained for the $1 / 4$ scale gear in its SILENCER reference configuration after transposition to full scale conditions according to Equs. (2) and (3). This comparison is depicted in Fig. 8 and shows a surprisingly good agreement except for a level peak at a frequency of about $1 \mathrm{kHz}$. It is interesting to note that both at model scale and at full scale a similar level peak occurs, but with a much higher level for the scale model gear. The comparison of broadband noise levels is excellent for forward arc radiation directions while, compared to the full scale build, the model gear turns out to be about 1 to $2 \mathrm{~dB}$ noisier for rear arc radiation directions. 


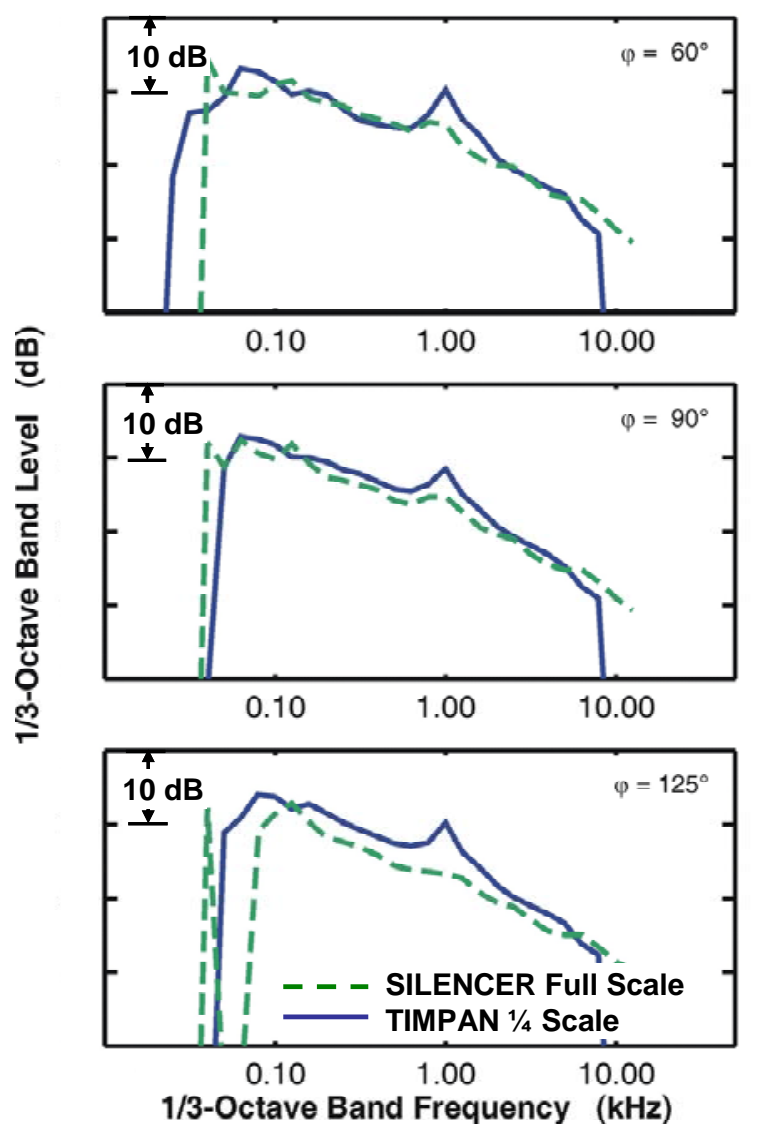

Figure 8. Comparison of normalized 1/3-oct. band level spectra for the TIMPAN $1 / 4$ scaled model gear in its SILENCER reference configuration with the spectrum for the original full scale SILENCER advanced gear for different polar radiation angles

As is obvious from this figure the chosen data reduction procedure (effect of speed presented for the reference configuration only) in the average provides reasonable results for all three radiation directions, i.e. in the forward arc $\left(\varphi_{\mathrm{x}}=60^{\circ}\right)$, corresponding to an aircraft in overhead position $\left(\varphi_{\mathrm{x}}=90^{\circ}\right)$, and into the rear arc $\left(\varphi_{\mathrm{x}}=125^{\circ}\right)$, respectively. Fig. 9 also shows that compared to the SILENCER reference configuration, a broadband noise reduction potential of up to about $10 \mathrm{~dB}$ is obtained for the quietest TIMPAN gear design. This noise reduction potential is most pronounced for the most important forward arc radiation direction. At very low Strouhal numbers the noise reduction potential is quite limited but still 2 to $4 \mathrm{~dB}$.

\section{Normalized noise level directivities}

From the comparison of the spectra presented above it is obvious that there is some Strouhal number effect on the noise directivity characteristics. Noise level directiv-
A further analysis of this apparent "tone" noise effect showed that this level peak scales on a Strouhal number basis and would correspond to a vortex shedding frequency from the dressings, which were simulated in TIMPAN by electric wire material. Any noise reduction potential derived from the $1 / 4$ scale model reference gear therefore must account for this particular model effect.

\section{A. Noise Characteristics}

1. Normalized noise level spectra

In Fig. 9 noise spectra are shown for the SILENCER reference configuration and the quietest TIMPAN configuration, respectively. This latter gear configuration combines the following features:

- Negative bogie angle (toe down),

- Narrow wheel spacing (both forward and rear wheel sets),

- Porous bogie and torque link fairings,

- TIMPAN brake fairings,

- $\quad$ Articulated TIMPAN door with $45^{\circ}$ ramp and

- $\quad$ TIMPAN side-stay design.

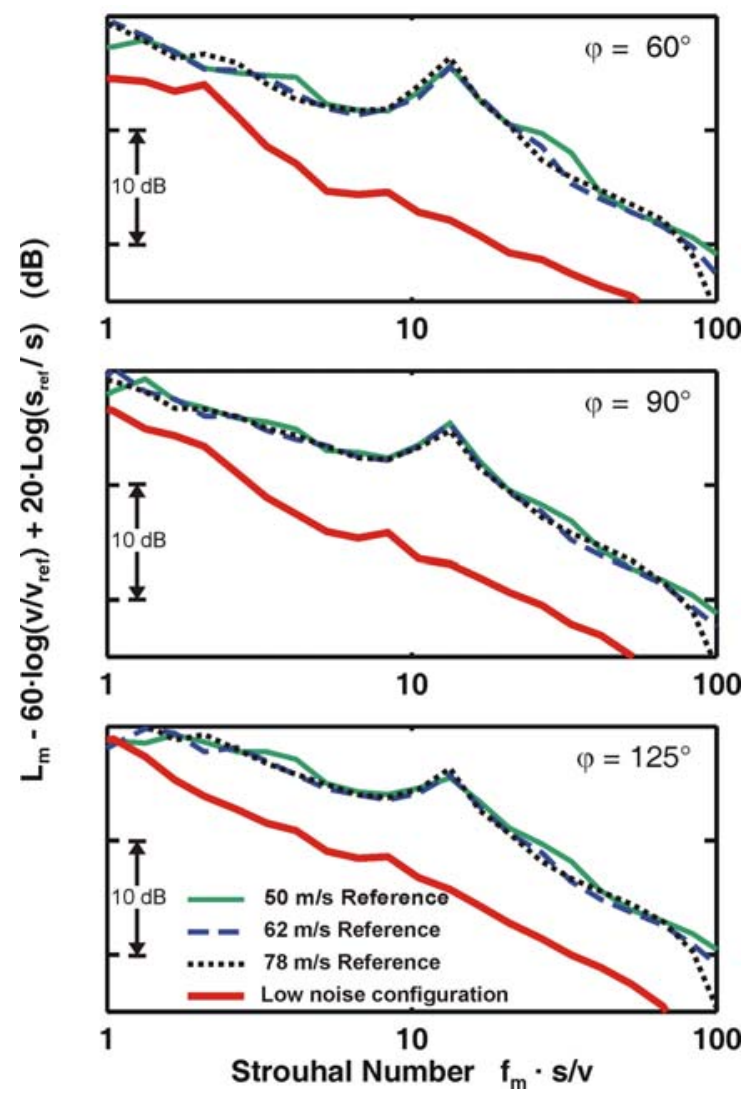

Figure 9. Comparison of normalized 1/3-oct. band noise spectra for the SILENCER reference and the TIMPAN low noise configuration for different polar radiation angles 


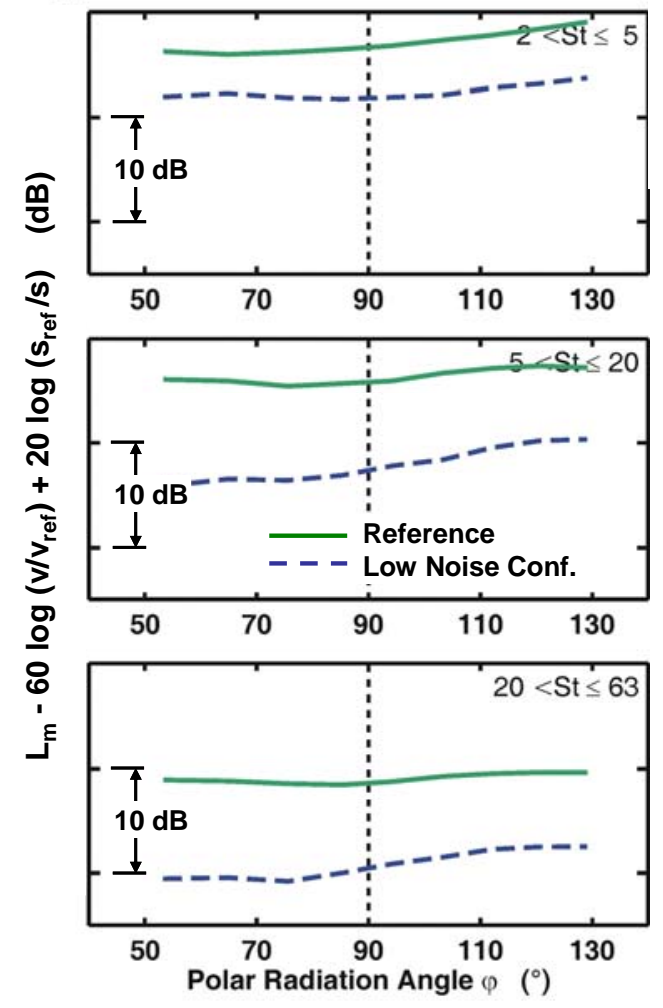

Figure 10. Comparison of polar directivities of normalized 1/3-oct. band noise levels in different bands of Strouhal number for the SILENCER reference and the TIMPAN low noise configuration

these graphs the solid black line indicates the position of the wall. While for the SILENCER reference configuration (upper part of Figure 11) the bogie area can be identified as the predominant noise source, with the gear in its low noise configuration (lower part of Figure 11) the highest noise levels originate from the leg-door/ side-stay area (Note: maximum levels are much lower for ID 55). From these results the conclusion can be derived that the TIMPAN low noise design was successful in dramatically reducing bogie related noise sources but needs further attention regarding an effective low noise design of the leg-door/ side-stay structure.

This conclusion is supported by the results from sideline measurements. Fig. 12 present a comparison of normalized landing gear noise directivities under the line of flight and for both outboard and inboard sideline radiation directions, respectively, for the SILENCER reference configuration and the TIMPAN low noise configuration. Note that for the SILENCER reference ities will therefore be presented for three different Strouhal number ranges. Sound energy is respectively integrated in the three ranges: $2<\mathrm{St} \leq 5 ; 5<\mathrm{St} \leq 20$ and $20<\mathrm{St} \leq 63$.

This normalized data representation inherently accounts for small differences in actual test speeds. Corresponding examples of noise directivities are presented in Fig. 10 for the SILENCER reference configuration and the TIMPAN low noise configuration, respectively. While the SILENCER configuration exhibits an almost omnidirectional noise radiation characteristic for the whole Strouhal number range of interest, the corresponding directivities for the low noise configuration feature an increasing level gradient with increasing Strouhal number, i.e. low levels in the forward arc and high levels in the rear arc radiation direction. Accordingly the TIMPAN low noise configuration provides an increasing noise reduction potential for higher Strouhal numbers and forward arc radiation direction.

In an attempt to explain this change in the respective noise level directivities, array source plots can be inspected. Most interesting results are provided by the microphone array presenting source distributions from a sideline view (Fig. 11). In

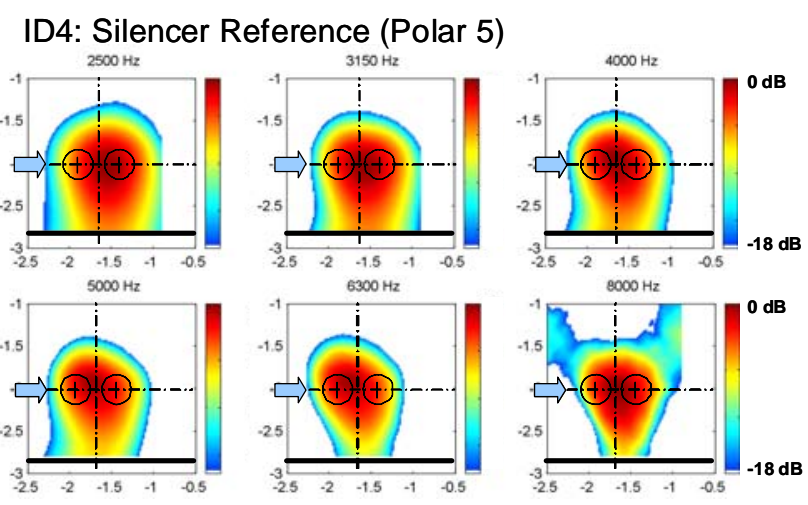

ID55: Low Noise Configuration (Polar 44)
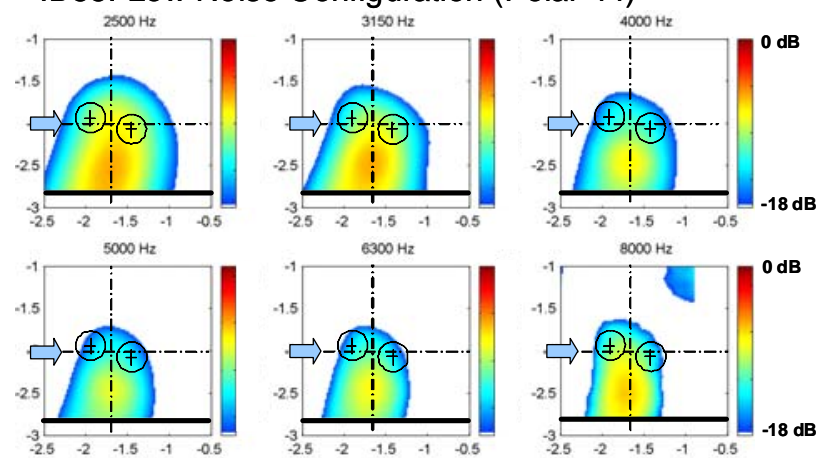

Figure 11. Comparison of noise source distributions from a "side view" for the SILENCER reference configuration and the TIMPAN low noise configuration and different $1 / 3-o c t$. band frequencies, respectively (wind speed from left to right; same level scales for the two configurations at identical frequency but autoscaling for different frequencies) 

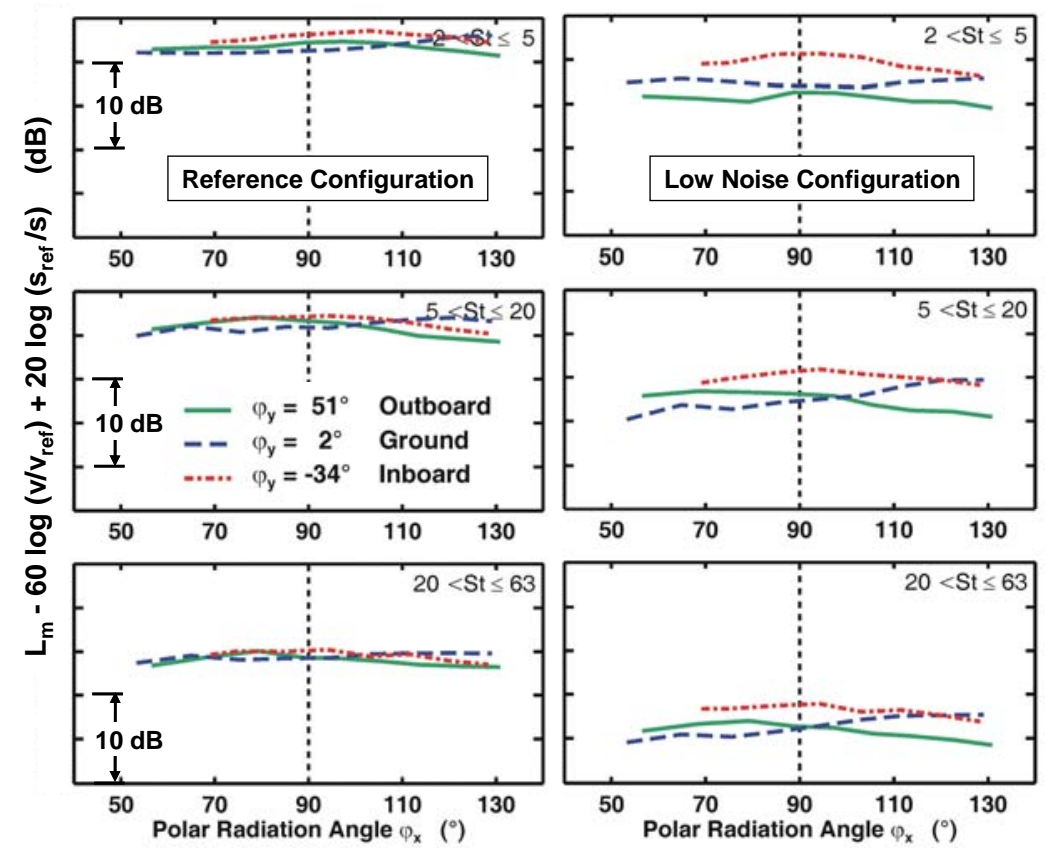

Figure 12. Polar directivities of non-dimensional 1/3-oct. band noise levels in different bands of Strouhal number for the SILENCER reference the TIMPAN low noise configurations and different azimuthal (sideline) radiation angles $\varphi_{\mathrm{y}}$, respectively configuration there is very little difference in the polar directivities for different sideline directions. However, differences show up for the quieter gear build as is presented in Fig. 12. It can be stated that the noise reduction obtained by this gear configuration is most pronounced for overhead ("ground") and outboard radiation directions, while the levels for inboard radiation direction remain relatively high. From the results as obtained for the variety of different gear configurations it turns out that this observation cannot be associated with any particular gear build but is specific for all relatively quiet configurations and thus supports the conclusion that the leg-door/ side-stay area is the remaining dominant source region compared to the bogie area for the SILENCER reference configuration. While the leg door shields noise to be radiated towards outboard directions, noise as generated by the various leg door con-

struction details on the inboard side of the door can freely radiate in this direction. From these results it may also be concluded, that noise originating from the bogie area features an almost omnidirectional directivity, while noise from the leg-door structure is more directional.

Any efforts to further reduce the noise radiation from this gear design thus obviously must focus on the leg-door/ side-stay components. However, this skewness of the azimuthal radiation directivity is not considered a problem with respect to aircraft noise impact on the ground because the presented directivities are referenced to a constant radiation radius. In a flyover situation noise levels experience a geometrical damping for sideline directions which would amount to about $3 \mathrm{~dB}$ for a $45^{\circ}$ azimuthal angle, thus compensating the observed "source" level increase shown in Fig. 12.

\section{B. Noise Reduction Potential}

The effectiveness of different noise reduction measures was determined through the computation of level differences from corresponding tests relative to a suitable reference configuration, i.e. the level difference from two gear configurations with one component change at a time.

From this exercise a rank order of noise reduction means' effectiveness is obtained, which however, to a limited extent suffers from the fact that not all these changes could be tested for the same reference configuration, meaning that a measure which was tested for a relatively noisy configuration will have a different effectiveness as it would have when tested on a relatively quiet configuration. Therefore no relevant rank order of priority can be provided. Still, some important general low noise design guidelines can be identified based on this procedure and will be discussed below.

\section{Bogie and torque link fairings}

The combined application of a bogie fairing and a torque link fairing (note: the torque link is installed in front of the gears' leg) seems to be a prerequisite for a low noise gear. It turned out that a porous design of both these fairings is an effective noise reduction feature. Fig. 13 depicts the individual effects on noise of these fairings for either a solid or porous make. For porous fairings, the flow displacement is significantly reduced while still achieving a low enough wake flow velocity.

In this context it should be mentioned that different wheel spacing did not have a notable effect on noise although this design parameter has some effect on the flow through the bogie structure. 

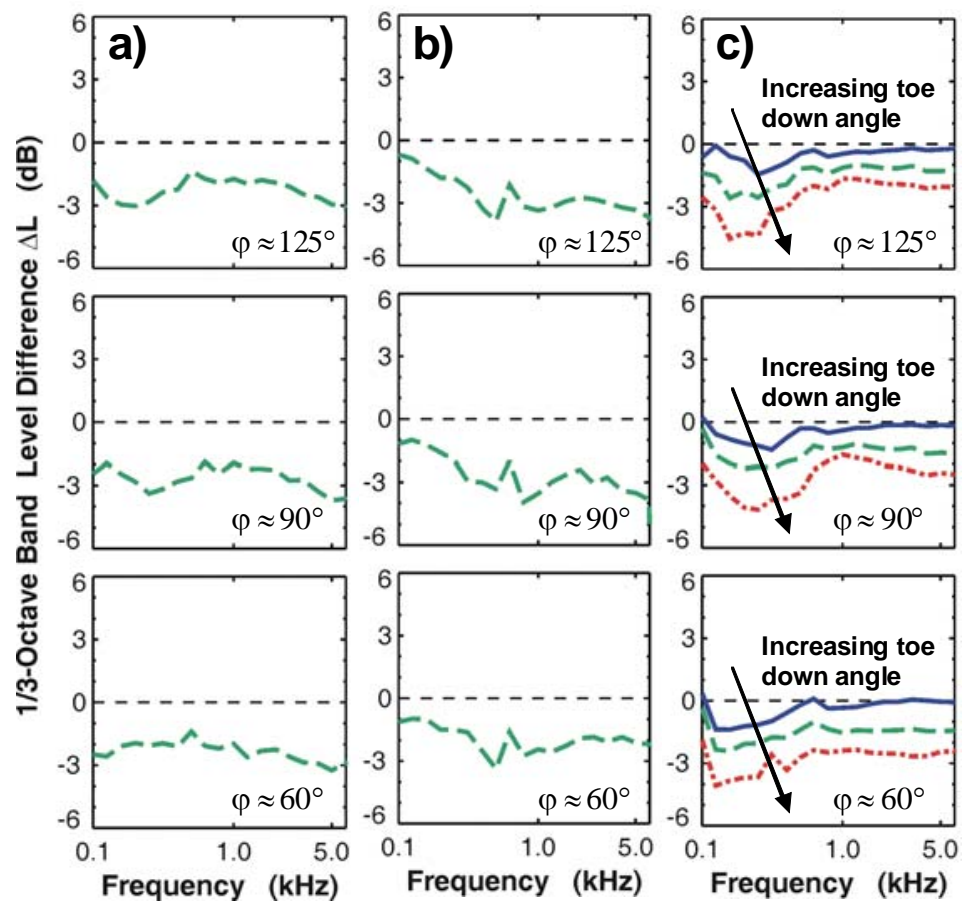

Figure 13. Effects of (a) porous bogie fairing and (b) porous torque link fairing w.r.t. solid fairings and (c) of bogie inclination angle

respect to noise. Such means are imperative because otherwise excess noise from the brakes will degrade the noise benefits from other noise reduction treatments.

\section{Leg-door/side-stay design}

The TIMPAN side-stay in combination with the articulated leg-door design turned out to be reasonably quiet but not as good as the SILENCER telescopic side-stay. This situation can significantly be improved by application of a ramp to reduce high frequency noise originating from the upper leg area and the sidestay joints and avoids flow interaction with the cavity aperture.

As a final result in this study the optimal combination of all tested gear modifications (see Section A for configuration details) provided a noise reduction of $8 \mathrm{~dB}(\mathrm{~A})$ in terms of the OASPL (Overall Sound Pressure Level) compared to the noise signature of the SILENCER reference configuration. The highest noise reduction potential is achieved for high frequencies and forward arc radiation direction (Fig. 14). The latter is the most important radiation direction, because the effect of convective amplification will enhance the noise levels in this radiation direction once this "stationary source noise characteristic" will be transposed to flight conditions.

\section{Noise Prediction}

Prior to the tests predictions of some of the key configurations were made using a semi-empirical landing gear noise prediction model [13]. Carrying out predictions prior to testing is beneficial as it confirms that the tests will provide useful results and guides the priorities for the experimental programme. Comparing predictions with measured data after the test is obviously beneficial for validating the model, but also helps in interpreting and extrapolating from the data.
4. Bogie inclination angle in combination with a bogie fairing

A successive increase in toe down bogie angle led to a successive decrease in noise (see Fig. 13). However, the noise-wise optimal toe down bogie angle might not have been captured in TIMPAN. This parameter needs a dedicated optimization cycle, keeping in mind that the final optimal angle might only be relevant for the gear architecture under consideration. It is assumed that the effect on noise of this design parameter is due to a shielding effect of a front wheel bogie fairing with respect to the flow impact at the rear axle.

5. Brake fairings

The type of brake fairings tested in TIMPAN on partially recessed brakes are considered an optimal solution with

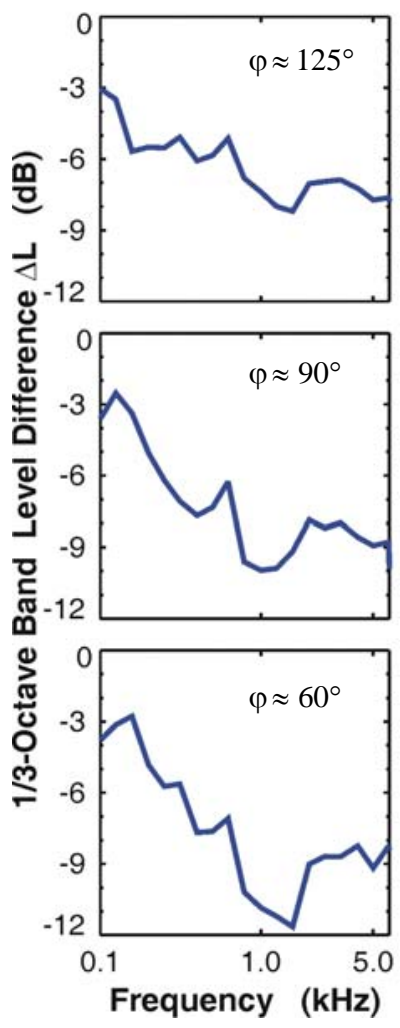

Figure 14. Noise reduction potential vs full scale frequency of the TIMPAN low noise configuration in comparison with the SILENCER reference configuration 
The prediction model has been validated in a considerable number of previous tests, but a major difficulty here was that the gear modifications to be tested (e.g. variation of wheel layout and bogie angle, ramp fairing, etc.) were expected to cause major changes to the flow distribution. As no CFD predictions were available it was necessary to

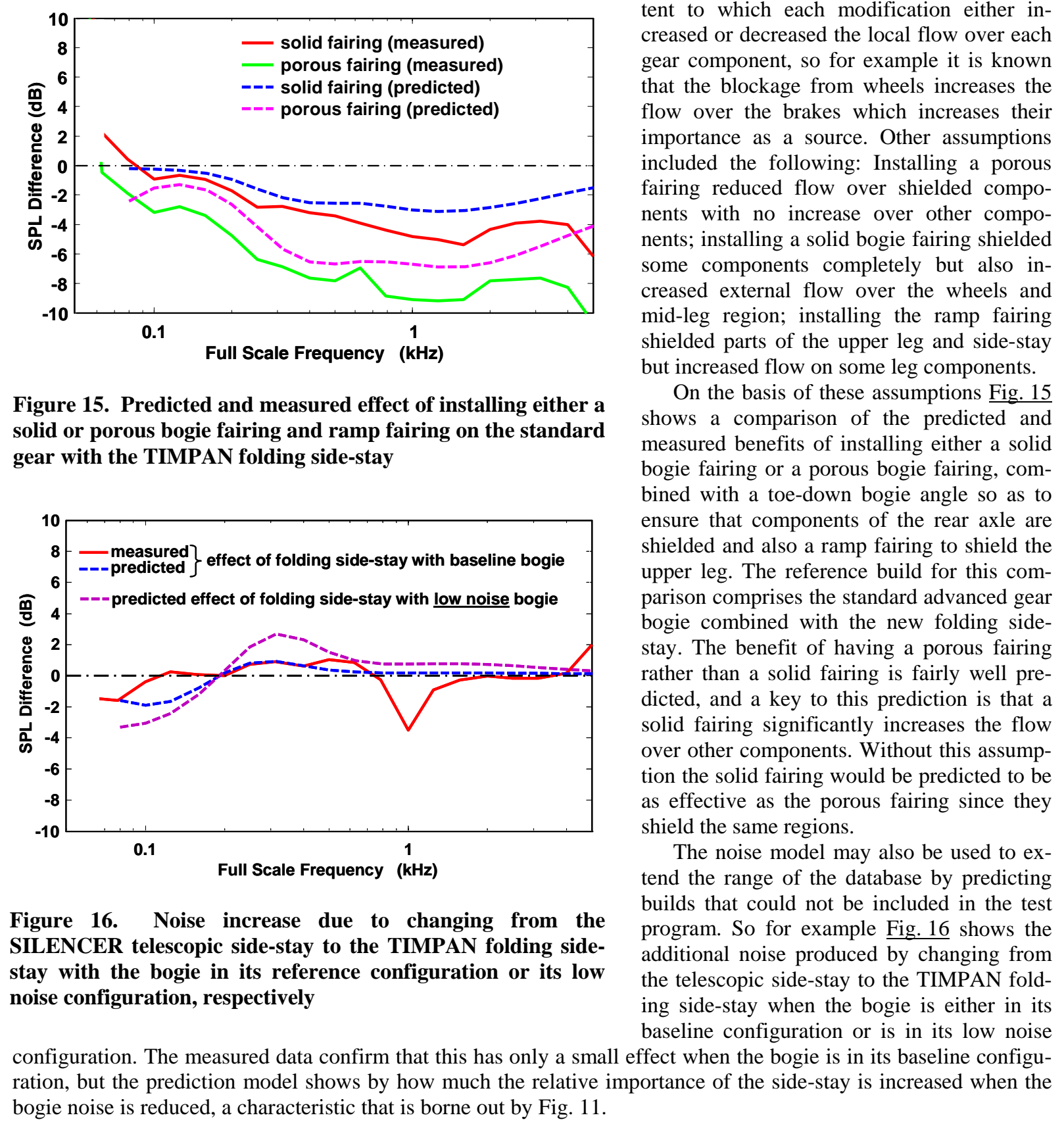

Figure 15. Predicted and measured effect of installing either a solid or porous bogie fairing and ramp fairing on the standard gear with the TIMPAN folding side-stay

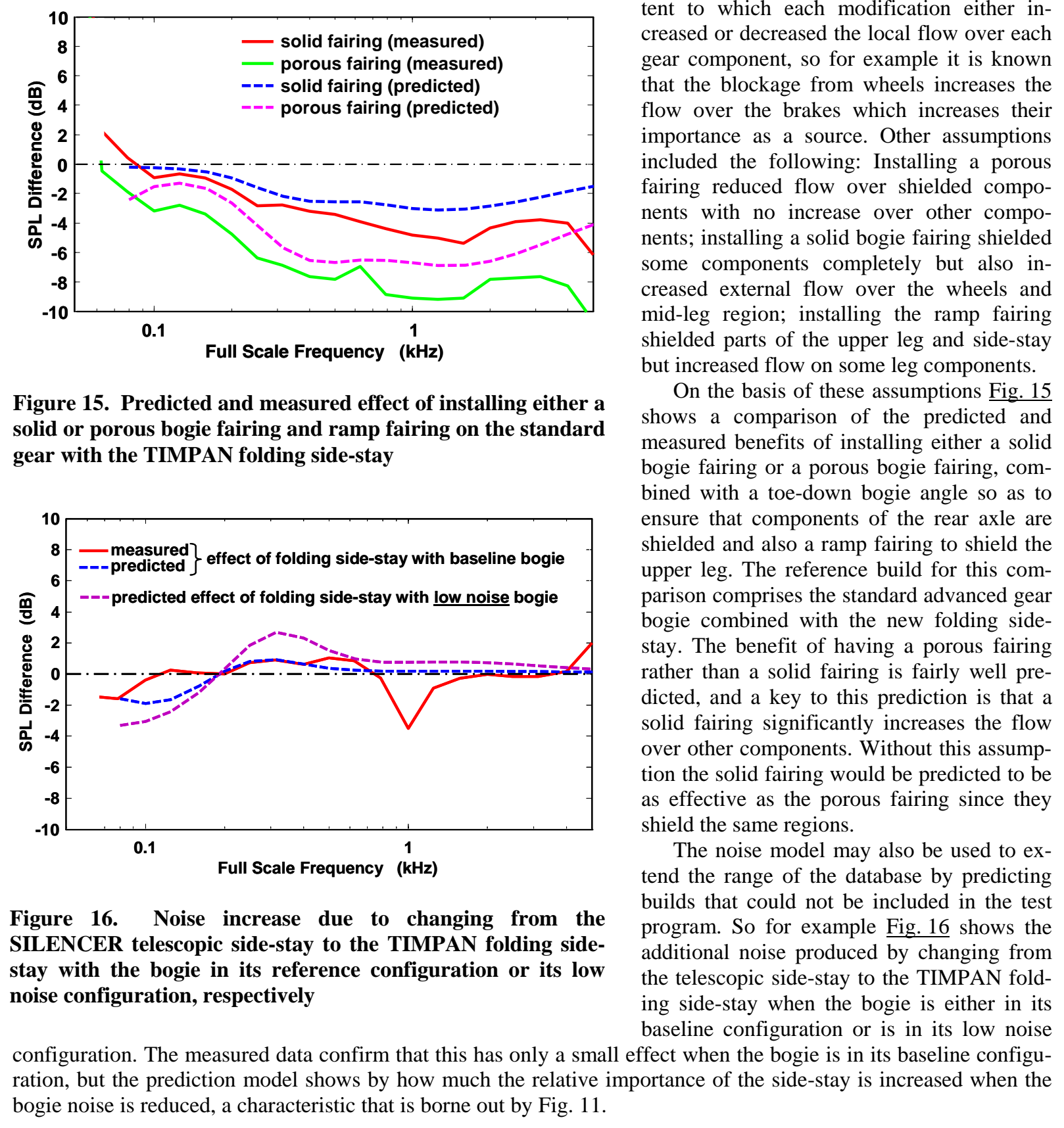

Figure 16. Noise increase due to changing from the SILENCER telescopic side-stay to the TIMPAN folding sidestay with the bogie in its reference configuration or its low noise configuration, respectively

configuration. The measured data confirm that this has only a small ration, but the prediction model shows by how much the relative importance of the side-stay is increased when the bogie noise is reduced, a characteristic that is borne out by Fig. 11.

\section{Transposition of Noise Data to Flight Conditions}

Measured noise data were finally transposed to flight conditions to estimate the potential impact of the new main landing gear design on the noise certification level (Effective Perceived Noise Levels) in approach for a generic long range twin engine aircraft. The low noise main landing gear configuration evaluated in-flight combines the following features: tent to which each modification either increased or decreased the local flow over each gear component, so for example it is known thew over the from wheels increases the importance as a source. Other assumptions included the following: Installing a porous fairing reduced flow over shielded components with no increase over other compoents; installing a solid bogie fairing shielded some components completely but also increased external flow over the wheels and shielded parts of the upper leg and side-stay but increased flow on some leg components.

On the basis of these assumptions Fig. 15 shows a comparison of the predicted and measured benefits of installing either a solid bogie fairing or a porous bogie fairing, comwith a toe-down bogie angle so as to ensure that components of the rear axle are upper leg. The reference build for this comparison comprises the standard advanced gear bogie combined with the new folding sidestay. The benefit of having a porous fairing rather than a solid fairing is fairly well predicted, and a key to this prediction is that a solid fairing significantly increases the flow over other components. Without this assumption the solid fairing would be predicted to be as effective as the porous fairing since they shield the same regions.

The noise model may also be used to extend the range of the database by predicting builds that could not be included in the test program. So for example Fig. 16 shows the additional noise produced by changing from the telescopic side-stay to the TIMPAN folding side-stay when the bogie is either in its baseline configuration or is in its low noise

make a number of assumptions about the ex-

10

American Institute of Aeronautics and Astronautics 092407 
- $\quad-15^{\circ}$ bogie angle (toe down),

- Narrow wheel spacing (both forward and rear wheel sets),

- Porous bogie and torque link fairings,

- TIMPAN brake fairings,

- Articulated TIMPAN door with $45^{\circ}$ ramp and

- TIMPAN side-stay design.

The estimation of approach noise levels is based on Airbus' total aircraft noise prediction code, accounting for landing gear source noise reduction in terms of level differences obtained from the wind tunnel tests after transposition to full scale conditions. This prediction code has been validated previously through comparisons with flight test data [14]. The code predicts the levels from each airframe and engine noise source separately.

The following parameters have been taken into account to conduct noise impact calculations for a long range twin engine aircraft configuration as defined in TIMPAN:

- Landing gear configurations: A340 baseline reference landing gears or with SILENCER advanced nose and main landing gear design [10];

- Aircraft configuration: $23^{\circ}$ slat, $32^{\circ}$ flap and landing gears down;

- Conventional approach trajectory: $-3^{\circ}$ glide slope;

- Flight parameters: Speed $\mathrm{V}_{\mathrm{C}}=145 \mathrm{kts}$, aircraft angle of attack $=3^{\circ}$;

At this stage neither a potential impact on the aerodynamic aircraft performance nor on aircraft weight was taken into account for this noise estimation.

Fig. 17 presents the estimated impact on the EPNL for the TIMPAN low noise main landing gear design in comparison with the SILENCER advanced gear design and the A330/A340 baseline main landing gear design, respectively, on (i) main landing gear source noise level, (ii) total landing gear source noise level, (iii) airframe noise, and (iv) total aircraft noise level. Accordingly, A340 main landing gear noise is reduced by more than $7 \mathrm{EPNdB}$ due to the TIMPAN low noise features. This corresponds to an additional $5 \mathrm{EPNdB}$ noise reduction in comparison with the previously developed advanced SILENCER main landing gear design [10].

With TIMPAN low noise main

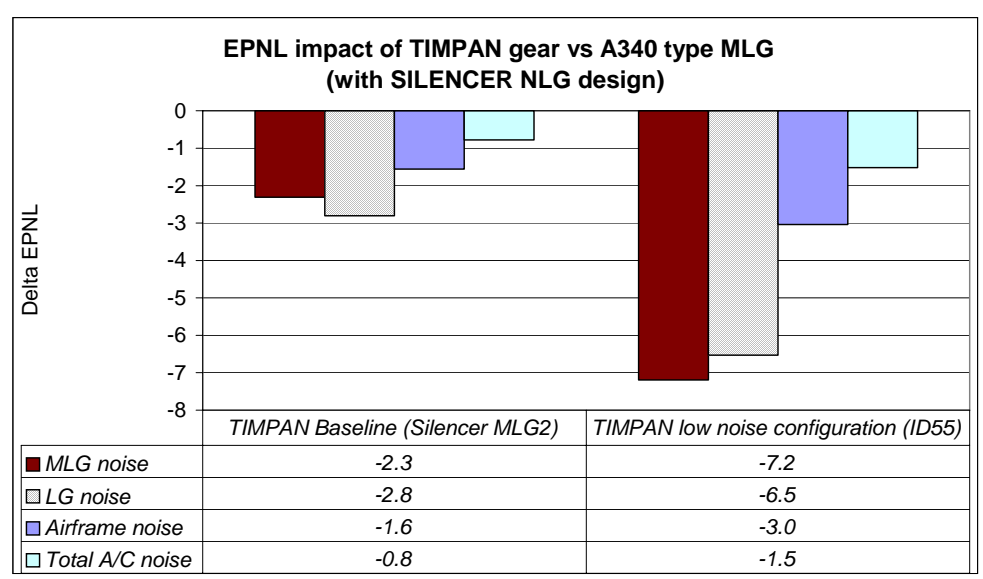

Figure 17. Impact on EPNL for approach conditions of the TIMPAN low noise main landing gear design vs the SILENCER gear design and the original A330/A340 gears, respectively

landing gears the overall landing gear

noise (including SILENCER nose landing gear design) is reduced by 6.5 EPNdB when compared to A330/A340 original landing gear noise levels. This demonstrates that the objectives of the TIMPAN research project to reduce landing gear noise levels by 6 EPNdB relative to the year 2000 technology has been achieved.

Finally, total aircraft noise can be reduced by 1.5 EPNdB in approach when applying the TIMPAN low noise features on A330/A340 main landing gears for otherwise identical noise levels related to both high-lift devices and the engines.

\section{Summary}

In the European co-financed research project TIMPAN the "advanced low noise design" of an A340 type 4wheel main landing gear, as developed in the former European SILENCER project, was further investigated with the objective to develop gear components which are as quiet or even quieter as the SILENCER design but with less weight penalty. A number of design options were developed to fit on the existing $1 / 4$ scale SILENCER advanced gear for noise testing in DNW-LLF. A variety of different gear configurations were tested for two wind speeds, respectively, including a new side-stay design, different modifications of bogie inclination (toe down), different wheel spacing, bogie fairings with different flow transparency, different leg-door and brake designs. 
Acoustic farfield data were acquired for frequencies up to $40 \mathrm{kHz}$ to account for the model scale factor. A comparison of normalized noise spectra and directivities as obtained for the SILENCER reference configuration at model scale with the spectra from the original SILENCER full scale test shows reasonable agreement.

An optimal combination of all tested gear modifications led to a noise reduction of up to $8 \mathrm{~dB}(\mathrm{~A})$ in terms of overall A-weighted noise levels relative to the SILENCER reference gear configuration. The main contributions to this noise reduction originated from an increase in toe down bogie inclination angle, both a bogie and torque link porous fairing in combination with a low noise side-stay and brake design and an alternative leg-door design (re SILENCER) in combination with a ramp.

In contrast to the SILENCER reference configuration the polar noise directivity for the TIMPAN low noise configuration is characterized by maximum high frequency noise levels in the rear arc radiation direction. Source location with microphone arrays and supported by the analysis of sideline noise level directivities, proved the leg-door/ side-stay structure to represent the dominating noise source area for the TIMPAN low noise main landing gear configuration, while noise originating from the bogie area was dominating for the SILENCER reference gear configuration.

The test data are broadly in line with predictions made using a semi-empirical prediction model, although the use of this model is limited by the lack of accurate information about the changes in local flow over each component.

Finally, measured noise data were transposed to flight conditions to estimate the overall approach noise reduction for a generic long range aircraft. The TIMPAN low noise main landing gear configuration provides more than 7 EPNdB main landing gear source noise reduction, which results in a total aircraft noise reduction of $1.5 \mathrm{EPNdB}$ for otherwise unchanged high-lift devices and engine noise levels.

\section{Acknowledgments}

The authors thank the staff of Airbus, BAE-Systems, Messier-Dowty, Messier-Bugatti and Southampton University for their efforts in manufacturing of landing gear components and their support during the test conduct. The acoustic data acquisition was prepared and conducted by DNW. The engaged support by the DNW project coordinator, Hermann Holthusen, is highly appreciated. Finally EC co-funding of this research project is also acknowledged.

\section{References}

${ }^{1}$ Dobrzynski, W. and Buchholz, H., "Full-Scale Noise Testing on Airbus Landing Gears in the German Dutch Wind Tunnel”, AIAA/CEAS 1997-1597, Atlanta/USA, 1997.

${ }^{2}$ Stoker, R.W., “Landing Gear Noise Test Report”, NASA Contract NAS1-97040, 1997.

${ }^{3}$ Dobrzynski, W., Chow, L. C., Guion, P., Shiells, D., "Research into Landing Gear Airframe Noise Reduction”, AIAA/CEAS Meeting Paper 2002-2409, Breckenridge/CO, 2002.

${ }^{4}$ Ravetta, P.A., Burdisso, R.A. and Ng, W.F., "Wind Tunnel Aeroacoustic Measurements of a 26\%-scale 777 Main Landing Gear Model”, AIAA/CEAS 2004-2885, Manchester/UK, 2004.

${ }^{5}$ Abeysinghe, A. et al., "QTD 2 (Quiet Technology Demonstrator) Main Landing Gear Noise Reduction Fairing Design and Analysis”, AIAA/CEAS 2007-3456, Rom/Italy, 2007.

${ }^{6}$ Remillieux, M.C. et al., "Noise Reduction of a Model-Scale Landing Gear Measured in the Virginia Tech Aeroacoustic Wind Tunnel”, AIAA/CEAS 2008-2818, Vancouver/Canada, 2008.

${ }^{7}$ Smith, M.G. et al., "Control of Noise Sources on Aircraft Landing Gear Bogies”, AIAA/CEAS 2006-2626, Cambidge/USA, 2006.

${ }^{8}$ Ravetta, P.A., Burdisso, R.A. and Ng, W.F., “Noise Control of Landing Gears Using Elastic Membrane-Based Fairings”, AIAA/CEAS 2007-3466, Rom/Italy, 2007.

${ }^{9}$ Boorsma, K,, Zhang, X. and Molin, N., "Perforated Fairings for Landing Gear Noise”, AIAA/CEAS 2008-2961, Vancouver/Canada, 2008.

${ }^{10}$ Dobrzynski, W. et al., "Design and Testing of Low Noise Landing Gears”, Journal of Aeroacoustics, Volume 5, Number 3, 233-262, 2006.

${ }^{11}$ Amiet, R. K., "Correction of Open Jet Wind Tunnel Measurements for Shear Layer Refraction", AIAA Meeting Paper 75532, Hampton, VA./USA, March 24-26, 1975.

${ }^{12}$ Bass, H. E., Sutherland, L. C., Zuckerwar, A. J., "Atmospheric Absorption of Sound: Update", J. Acoust. Soc. Am. 88(4), pp. 2019-2021, Oct. 1990.

${ }^{13}$ Smith, M. G. and Chow, L. C., "Validation of a Prediction Model for Aerodynamic Noise from Aircraft Landing Gears", AIAA/CEAS 2002-2581, Breckenridge/USA, 2002.

${ }^{14}$ Molin, N. et al, "Prediction of low noise aircraft landing gears and comparison with test results", AIAA/CEAS 2006- 2623, Cambridge/USA , 2006. 